\title{
ANALYSIS OF A POSSIBLE BATTERY CHARGERTOPOLOGY
}

Battery chargers are very important, especially for handhelds and cars. In this paper a converter is analyzed which enables us to control the voltage across the battery for a high input voltage range. Using the converter as a battery charger, the load current has to be controlled. Consequently, a two-point controller can be used. Therefore, a draft of a two-point controller is given. In addition, the weighted state differential equations are linearized in order to use the possibilities of the linear control theory. A basic analysis of the charger topology is given, the losses are approximated, and some experimental results are presented.

Keywords: Battery charger, DC/DC converter, modelling.

\section{Introduction}

The basic converter is shown in Fig. 1 [1] and consists of an inductor $L$, an inductor $L_{B}$, a capacitor $C$, an active switch $S$ and a passive switch $D$. The positive pole of the output voltage is the cathode of the diode. The battery is modelled by a voltage source $U_{B}$. Due to the additional capacitor $C$ and the additional inductor $L$, the classical converter, which is only a step-down converter, is transferred into a step-up-down converter. The mean value of the output voltage $U_{2}$, with the duty ratio of the active switch $d$ (the on-time of the active switch referred to the switching period) and neglecting the losses is

$$
U_{2}=\frac{d}{(1-d)} \cdot U_{1} .
$$

The converter is a step-up step-down converter, which also enables us to drive a DC motor in one direction (one-quadrant drive), controlled braking is not possible with this circuit. For other concepts, control applications, and detail about the electrical machine refer to the references [2] till [7].

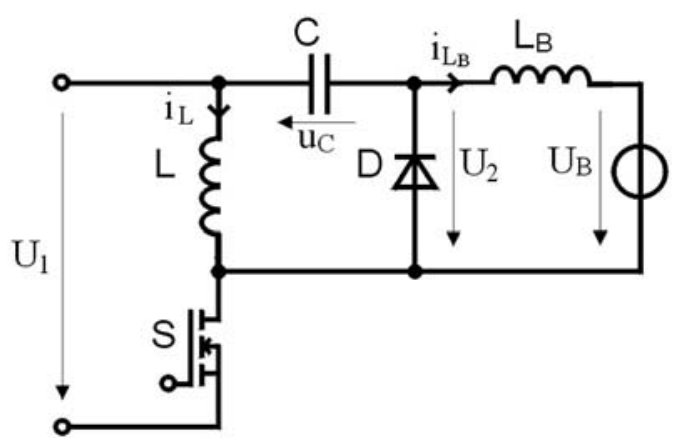

Fig. 1 Step-up-down converter

\section{Basic analysis}

The basic analysis has to be done with idealized components (that means no parasitic resistors, no switching losses) and for the continuous mode in steady (stationary) state. A good way to start is to consider the voltage across the inductors.

Since for the stationary case the absolute values of the voltagetime-areas of the inductors have to be equal (the voltage across the inductor has to be zero in the average), we can easily draw the shapes according to Figs. 2 and 3. (Here the capacitor is assumed to be so large that the voltage can be regarded constant during a pulse period.)
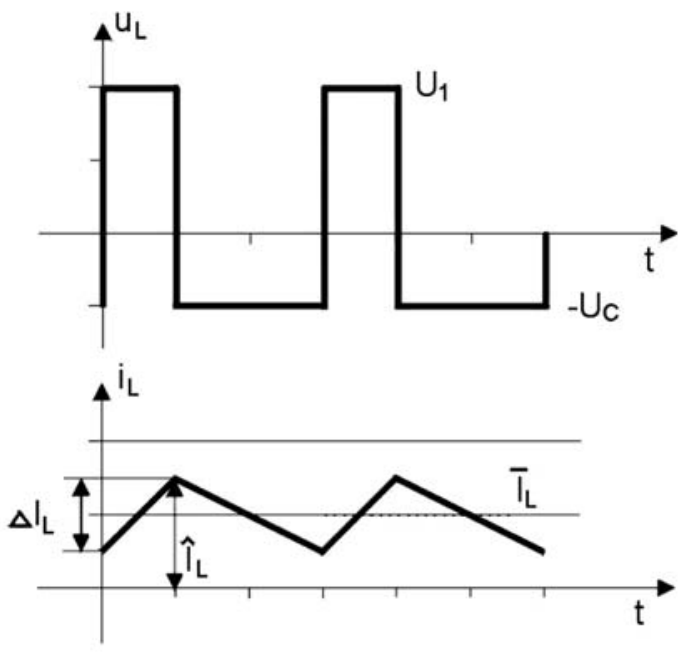

Fig. 2 Voltage across and current through the inductor $L$

\footnotetext{
* Yvonne Flicker, Felix A. Himmelstoss

University of Applied Science Technikum Wien, E-mail: Felix.Himmelstoss@technikum-wien.at
} 

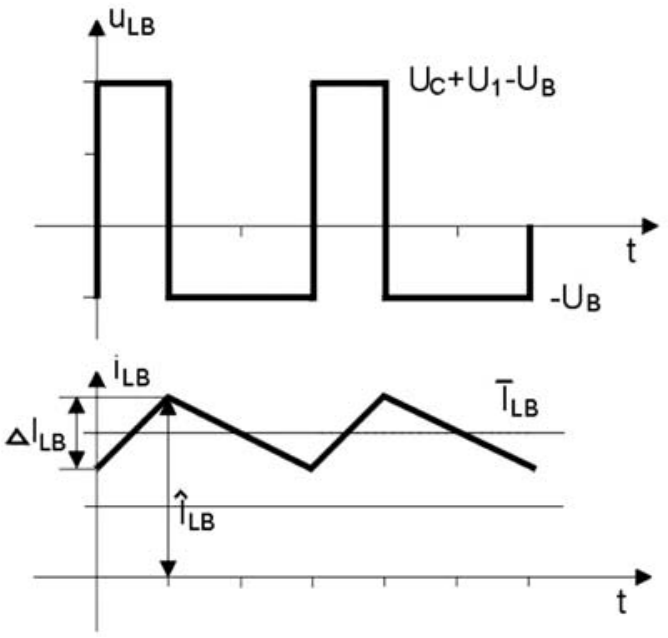

Fig. 3 Voltage across and current through the inductor $L_{B}$

Figure 2 shows the current through and the voltage across inductor $L$. The current rate of rise of course depends on the values of $L$ and $U_{1}$. Figure 3 shows the current through and the voltage across inductor $L_{B}$. Based on the equality of the voltage-time-areas in the stationary case, it is easy to give the transformation relationship for the battery voltage $U_{B}$ as a function of the input voltage $U_{1}$ and the duty ratio $d$. From Fig. 3 we get

$$
\left(U_{C}+U_{1}-U_{B}\right) \cdot d=U_{B} \cdot(1-d)
$$

and from Fig. 2

$$
U_{1} \cdot d=U_{c} \cdot(1-d) .
$$

After a few steps we get the voltage transformation law

$$
M=\frac{U_{B}}{U_{1}}=\frac{d}{(1-d)} \text {. }
$$

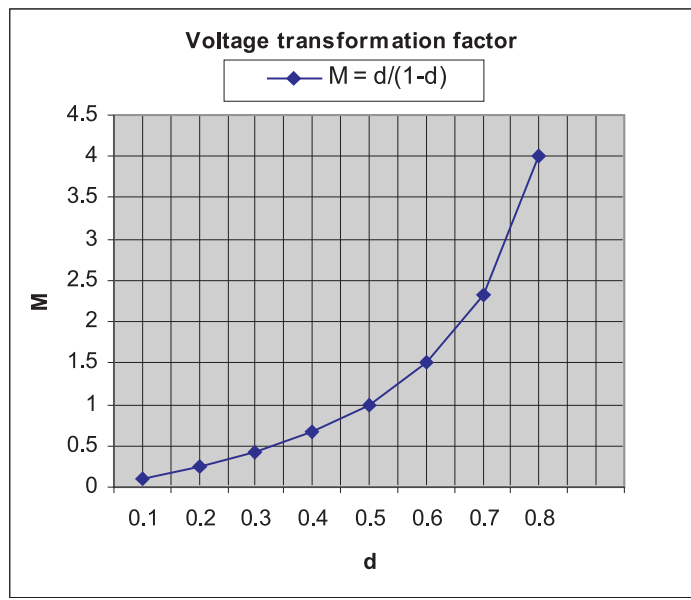

Fig. 4 Voltage transformation factor in dependence on the duty cycle
Figure 4 shows the voltage transformation factor $M=\frac{U_{B}}{U_{1}}$ (the ratio of mean value of the output voltage of the converter to input voltage) which is dependant on the duty cycle $d$. The converter is a step-up-down converter.

In the same manner a relationship for the currents through the inductors can be derived based on the equality of the absolute values of the current-time-areas of the capacitor during on- and offtimes of the active switch. From Fig. 5, which shows the current through the capacitor $C$, we get

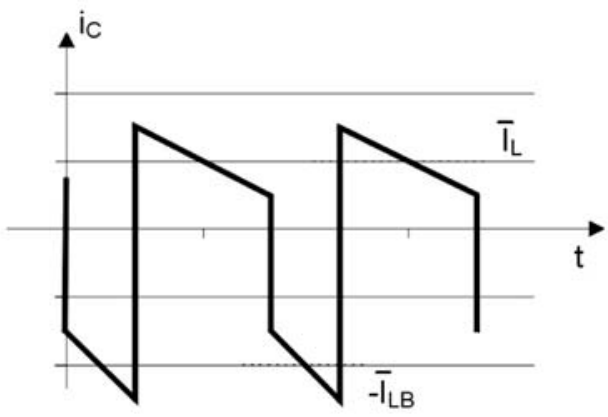

Fig. 5 Current through the capacitor $C$

$\bar{I}_{L} \cdot(1-D)=\bar{I}_{L B} \cdot d$

$\bar{I}_{L}=\bar{I}_{L B} \cdot \frac{d}{(1-d)}$.
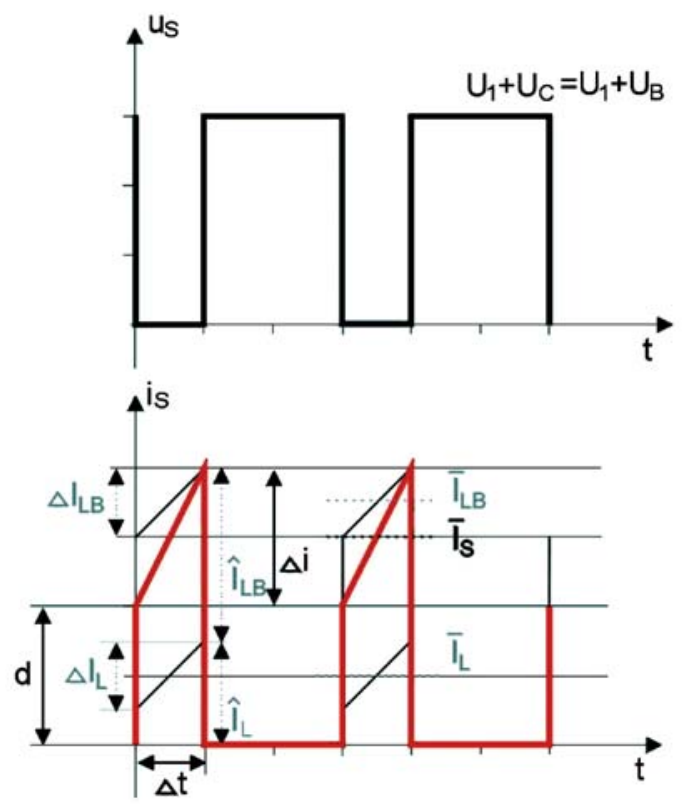

Fig. 6 Voltage across and current through the switch $S$

14 COMMUNICATIONS 3/2013 
From the schematic Fig. 1 one can immediately see that the current through the semiconductor devices is the sum of the inductor currents $i_{L}$ and $i_{L B}$ (through the switch $S$ during $T_{\text {on }}=d \cdot T$ through the diode $D$ during $T_{\text {off }}=(1-\mathrm{d}) \cdot T$. Therefore, the current maximum values for the semiconductor devices are approximately

$$
\tilde{I}_{S, \text { max }}=\tilde{I}_{D, \max }=\bar{I}_{L}+\bar{I}_{L B} .
$$

Figure 6 shows the current through the active switch and Fig. 7 through the passive switch (diode).

From Fig. 6 one can see that the correct current through the switch $S$ is

$$
\begin{aligned}
& i_{S}=i_{L}+i_{L B}=\frac{\Delta i}{\Delta t} \cdot t+d= \\
& =\frac{\left(\Delta I_{L}+\Delta I_{L B}\right) \cdot t}{d \cdot T}+\bar{I}_{L}+\bar{I}_{L B}-\frac{\Delta I_{L}+\Delta I_{L B}}{2} .
\end{aligned}
$$

The rms values are important for the calculation of the onstate losses. One can calculate the exact $r m s$ value for switch $S$ according to

$$
I_{S, r m s}=\sqrt{\frac{1}{T} \cdot \int_{0}^{d \cdot T}\left(i_{S}\right)^{2} \cdot d t} .
$$

After a few steps we get the exact rms value for the switch $S$ to

$$
I_{S, r m s}=\sqrt{\left[\begin{array}{l}
\frac{\left(\Delta I_{L}+\Delta I_{L B}\right)^{2}}{3}+ \\
+\left(\Delta I_{L}+\Delta I_{L B}\right) \cdot\left(\bar{I}_{L}+\bar{I}_{L B}-\frac{\Delta I_{L}+\Delta I_{L B}}{2}\right)+ \\
+\left(\bar{I}_{L}+\bar{I}_{L B}-\frac{\Delta I_{L}+\Delta I_{L B}}{2}\right)^{2}
\end{array}\right] \cdot d} \cdot d
$$

The current $r m s$ value in the middle for the transistor $S$ with the current mean value of the inductors is approximately

$$
\bar{I}_{S, r m s}=\left(\bar{I}_{L}+\bar{I}_{L B}\right) \cdot \sqrt{d} .
$$

The conduction losses for the switch $S$ with $R_{D S(o n)}$ as on-resistance of the transistor $S$ can be calculated according to

$$
P_{v, S}=\frac{1}{T} \int_{0}^{d \cdot T} u_{D S} \cdot i_{S} \cdot d t=\frac{1}{T} \int_{0}^{d \cdot T} R_{D S(o n)} \cdot\left(i_{S}\right)^{2} \cdot d t .
$$

After a few steps we get for the conduction losses of the active switch $S$

$$
\begin{aligned}
& P_{v, S}=R_{D S(o n)} {\left[\begin{array}{l}
\frac{\left(\Delta I_{L}+\Delta I_{L B}\right)^{2}}{3}+ \\
+\left(\Delta I_{L}+\Delta I_{L B}\right) \cdot\left(\bar{I}_{L}+\bar{I}_{L B}-\frac{\Delta I_{L}+\Delta I_{L B}}{2}\right)+ \\
+\left(\bar{I}_{L}+\bar{I}_{L B}-\frac{\Delta I_{L}+\Delta I_{L B}}{2}\right)^{2}
\end{array}\right] \cdot d } \\
& P_{v, S}=R_{D S(\text { on })} \cdot I_{S, r \text { ms }}^{2}
\end{aligned}
$$

The conduction losses of transistor $S$ with (11) are approximately

$$
\begin{aligned}
& P_{v, S}=R_{D S(o n)} \cdot I_{S, r m s}^{2}=R_{D S(o n)} \cdot\left[\left(\bar{I}_{L}+\bar{I}_{L B}\right) \cdot \sqrt{d}\right]^{2} \\
& P_{v, S}=R_{D S(o n)} \cdot\left(\bar{I}_{L}+\bar{I}_{L B}\right)^{2} \cdot d
\end{aligned}
$$

which could be supplemented by the current ripple by using the exact equation (10).
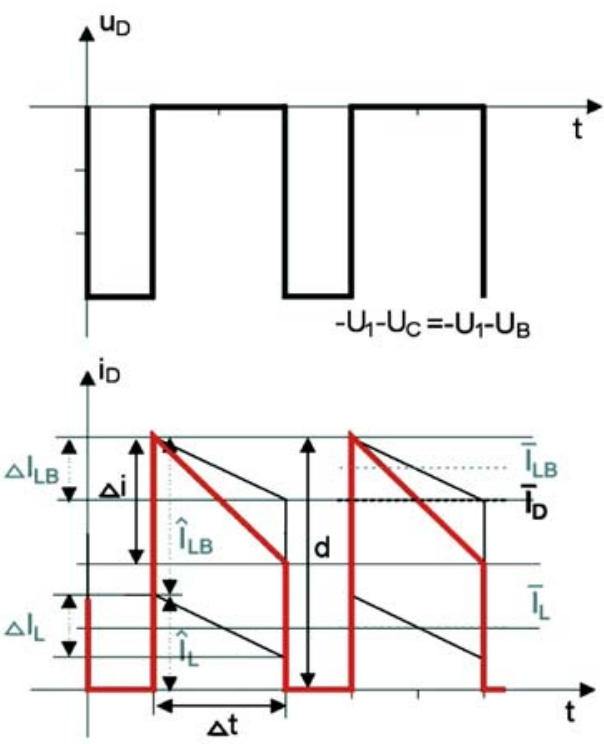

Fig. 7 Voltage across and current through the diode $D$

From Fig. 7 one can see that the current through the passive switch $D$ is

$$
\begin{aligned}
& i_{D}=i_{L}+i_{L B}=-\frac{\Delta i}{\Delta t} \cdot t+d= \\
& =-\frac{\left(\Delta I_{L}+\Delta I_{L B}\right) \cdot t}{(1-d) \cdot T}+\bar{I}_{L}+\bar{I}_{L B}+\frac{\Delta I_{L}+\Delta I_{L B}}{2}
\end{aligned}
$$

Therefore, one can calculate the exact $r m s$ value for diode $D$ according to

$$
I_{D, r m s}=\sqrt{\frac{1}{T} \cdot \int_{0}^{(1-d) \cdot T}\left(i_{D}\right)^{2} \cdot d t} .
$$

After a few steps we get the exact $r m s$ value for the diode

$$
I_{D, r m s}=\sqrt{\left[\begin{array}{l}
\frac{\left(\Delta I_{L}+\Delta I_{L B}\right)^{2}}{3}+ \\
+\left(\Delta I_{L}+\Delta I_{L B}\right) \cdot\left(\bar{I}_{L}+\bar{I}_{L B}-\frac{\Delta I_{L}+\Delta I_{L B}}{2}\right)+ \\
+\left(\bar{I}_{L}+\bar{I}_{L B}-\frac{\Delta I_{L}+\Delta I_{L B}}{2}\right)^{2}
\end{array}\right] \cdot(1-d) \cdot(17)}
$$


The current $r m s$ value in the middle for the diode $D$ with the current mean value of the inductors is approximately

$$
\bar{I}_{D, r m s}=\left(\bar{I}_{L}+\bar{I}_{L B}\right) \cdot \sqrt{(1-d)} .
$$

The conduction losses for the diode $D$ with $R_{D}$ as differential resistor of the diode and $U_{V D}$ as the fixed forward voltage can be calculated according to

$$
\begin{aligned}
& P_{v, D}=\frac{1}{T} \int_{0}^{(1-d) \cdot T} u_{D} \cdot i_{D} \cdot d t= \\
& =\frac{1}{T} \int_{0}^{(1-d) \cdot T}\left[U_{V D} \cdot i_{D}+R_{D} \cdot\left(i_{D}\right)^{2}\right] \cdot d t
\end{aligned}
$$

After a few steps we get the conduction losses for the passive switch $D$

$$
\begin{aligned}
& P_{v, D}=U_{V D} \cdot\left[\begin{array}{l}
-\frac{\Delta I_{L}+\Delta I_{L B}}{2}+ \\
+\left(\bar{I}_{L}+\bar{I}_{L B}-\frac{\Delta I_{L}+\Delta I_{L B}}{2}\right)
\end{array}\right] \cdot(1-d)+ \\
& +R_{D} \cdot\left[\begin{array}{l}
\frac{\left(\Delta I_{L}+\Delta I_{L B}\right)^{2}}{3}- \\
-\left(\Delta I_{L}+\Delta I_{L B}\right) \cdot\left(\bar{I}_{L}+\bar{I}_{L B}-\frac{\Delta I_{L}+\Delta I_{L B}}{2}\right) \\
+\left(\bar{I}_{L}+\bar{I}_{L B}-\frac{\Delta I_{L}+\Delta I_{L B}}{2}\right)^{2}
\end{array}\right] \cdot(1-d) \\
& P_{v, D}=U_{V D} \cdot\left[\begin{array}{l}
-\frac{\Delta I_{L}+\Delta I_{L B}}{2}+ \\
+\left(\bar{I}_{L}+\bar{I}_{L B}-\frac{\Delta I_{L}+\Delta I_{L B}}{2}\right)
\end{array}\right] . \\
& .(1-d)+R_{D} \cdot I_{D, r m s}^{2}
\end{aligned}
$$

The conduction losses of diode $D$ with (18) are approximately

$$
\begin{aligned}
& P_{v, D}=U_{V D} \cdot\left(\bar{I}_{L}+\bar{I}_{L B}\right) \cdot(1-d)+R_{D} \cdot I_{D, r m s}^{2} \\
& P_{v, D}=\left[U_{V D} \cdot\left(\bar{I}_{L}+\bar{I}_{L B}\right)+R_{D} \cdot\left(\bar{I}_{L}+\bar{I}_{L B}\right)^{2}\right] \cdot(1-d),
\end{aligned}
$$

which omits the current ripple.

So one can calculate the total conduction losses according to

$$
P_{v}=P_{v, S}+P_{v, D}
$$

We can calculate a rough approximation of the total conduction losses according to (22) and get

$$
\begin{aligned}
& P_{v}=\left[R_{D S(o n)} \cdot d+R_{D} \cdot(1-d)\right] \cdot\left(\bar{I}_{L}+\bar{I}_{L B}\right)^{2}+ \\
& +U_{V D} \cdot\left(\bar{I}_{L}+\bar{I}_{L B}\right) \cdot(1-d)
\end{aligned}
$$

One can calculate the efficiency of the converter (taking only the semiconductor conduction losses into account) according to

$$
\eta=\frac{P_{\text {out }}}{P_{\text {in }}}=\frac{\left(U_{B} \cdot \bar{I}_{L B}\right)}{\left(U_{B} \cdot \bar{I}_{L B}\right)+P_{v}} .
$$

It is therefore easy to calculate a rough approximation of the efficiency of the converter for continuous operation mode and we get

$$
\eta=\frac{1}{1+\frac{\left[R_{D S(a n)} \cdot d+R_{d} \cdot(1-d)\right] \cdot\left(\bar{I}_{L}+\bar{I}_{L B}\right)+U_{V D} \cdot\left(\bar{I}_{L}+\bar{I}_{L B}\right) \cdot(1-d)}{U_{B} \cdot \bar{I}_{L B}}}
$$

\section{Converter model}

The state variables are the inductor current $i_{L}$, the charging inductor current $i_{L B}$, and the capacitor voltage $u_{C}$. The input variables are the input voltage $u_{1}$ and the voltage $u_{B}$. The fixed forward voltage of the diode (the diode is modelled as a fixed forward voltage $U_{V D}$ and an additional voltage drop depending on the differential resistor of the diode $R_{D}$ ) is included as an additional vector. The parasitic resistances are the on-resistance of the active switch $R_{S}$, the series resistance of the converter coil $R_{L}$, the series resistance of the charging coil $R_{L B}$, the series resistor of the capacitor $R_{C}$, and the differential resistor of the diode $R_{D}$.

In continuous inductor current mode there are two states. In state one the active switch is turned on and the passive switch is turned off. Figure 8 shows this switching state one.

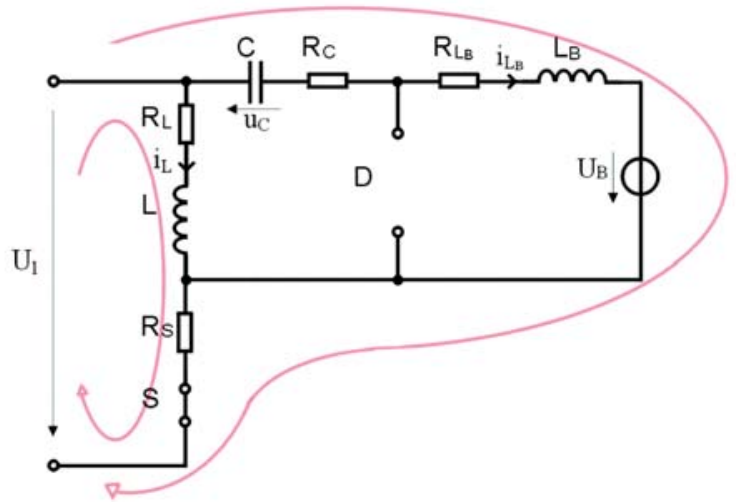

Fig. 8 Equivalent circuit for state one of the converter

The state space equations are now

$$
\begin{aligned}
& \frac{d i_{L}}{d t}=\frac{-i_{L} \cdot\left(R_{L}+R_{S}\right)-i_{L B} \cdot R_{S}+u_{1}}{L} \\
& \frac{d i_{L B}}{d t}=\frac{-i_{L} \cdot R_{S}-i_{L B} \cdot\left(R_{L B}+R_{C}+R_{S}\right)+u_{C}+u_{1}+u_{B}}{L_{B}} \\
& \frac{d u_{C}}{d t}=\frac{-i_{L B}}{C}
\end{aligned}
$$


leading to the state space description

$$
\frac{d}{d t}\left(\begin{array}{l}
i_{L} \\
i_{L B} \\
u_{C}
\end{array}\right)=\left[\begin{array}{ccc}
\frac{-\left(R_{L}+R_{S}\right)}{L} & \frac{-R_{S}}{L} & 0 \\
\frac{-R_{S}}{L_{B}} & \frac{-\left(R_{L B}+R_{C}+R_{S}\right)}{L_{B}} & \frac{1}{L_{B}} \\
0 & \frac{-1}{C} & 0
\end{array}\right] \cdot\left(\begin{array}{c}
i_{L} \\
i_{L B} \\
u_{C}
\end{array}\right)+\left[\begin{array}{cc}
\frac{1}{L} & 0 \\
\frac{1}{L_{B}} & \frac{-1}{L_{B}} \\
0 & 0
\end{array}\right] \cdot\left(\begin{array}{l}
u_{1} \\
u_{B}
\end{array}\right)
$$

In state two the active switch is turned off and the passive switch is turned on. Figure 9 shows this switching state two.

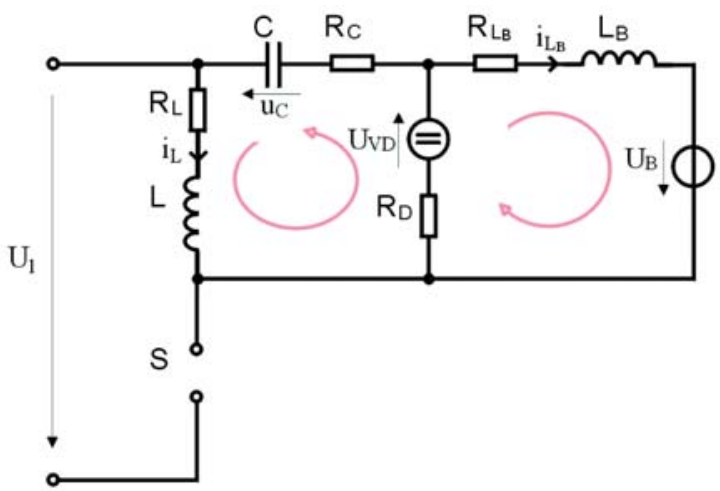

Fig. 9 Equivalent circuit for state two of the converter

The describing equations are

$\frac{d i_{L}}{d t}=\frac{-i_{L} \cdot\left(R_{L}+R_{C}+R_{D}\right)-i_{L B} \cdot R_{D}-U_{V D}-u_{C}}{L}$

$$
\begin{aligned}
\frac{d i_{L B}}{d t} & =\frac{-i_{L} \cdot R_{D}-i_{L B} \cdot\left(R_{L B}+R_{D}\right)-U_{V D}-u_{B}}{L_{B}} \\
\frac{d u_{C}}{d t} & =\frac{i_{L}}{C}
\end{aligned}
$$

leading to the state space description (33)

When using two active switches in push-pull mode (a second active switch instead of the diode), the diode is shunted and $U_{V D}$ can be set to zero. $R_{D}$ is then the on-resistance of the second switch. Combining the two systems by the state-space averaging method leads to a model, which describes the converter in the mean. We can combine these two sets of equations, providing that the system time constants are large compared to the switching period.

Weighed by the duty ratio, the combination of the two sets yields to (34)

The dynamic behavior of the idealized converter is described correctly in the average by the given system of equations, thus quickly giving us a general view of the dynamic behavior of the converter. The superimposed ripple (which appears very pronounced in the coils) is of no importance for qualifying the dynamic behavior. This model is also appropriate as large-signal model, because no limitations with respect to the signal values have been made.

$\frac{d}{d t}\left(\begin{array}{l}i_{L} \\ i_{L B} \\ u_{C}\end{array}\right)=\left[\begin{array}{ccc}\frac{-\left(R_{L}+R_{C}+R_{D}\right)}{L} & \frac{-R_{D}}{L} & \frac{-1}{L} \\ \frac{-R_{D}}{L_{B}} & \frac{-\left(R_{L B}+R_{D}\right)}{L_{B}} & 0 \\ \frac{1}{C} & 0 & 0\end{array}\right] \cdot\left(\begin{array}{c}i_{L} \\ i_{L B} \\ u_{C}\end{array}\right)+\left[\begin{array}{cc}0 & 0 \\ 0 & \frac{-1}{L_{B}} \\ 0 & 0\end{array}\right] \cdot\left(\begin{array}{l}u_{1} \\ u_{B}\end{array}\right)+\left[\begin{array}{c}\frac{-1}{L} \\ \frac{-1}{L_{B}} \\ 0\end{array}\right] \cdot u_{V D}$

$\frac{d}{d t}\left(\begin{array}{c}i_{L} \\ i_{L B} \\ u_{C}\end{array}\right)=\left[\begin{array}{ccc}\frac{-\left[d \cdot R_{S}+(1-d) \cdot\left(R_{C}+R_{D}\right)+R_{L}\right]}{L} & \frac{-\left[d \cdot R_{S}+(1-d) \cdot R_{D}\right]}{L} & \frac{(d-1)}{L} \\ \frac{-\left[d \cdot R_{S}+(1-d) \cdot R_{D}\right]}{L_{B}} & \frac{-\left[d \cdot\left(R_{C}+R_{S}\right)+R_{L B}+(1-d) \cdot R_{D}\right]}{L_{B}} & \frac{d}{L_{B}} \\ \frac{(1-d)}{C} & \frac{-d}{C} & 0\end{array}\right]$.

$\cdot\left(\begin{array}{l}i_{L} \\ i_{L B} \\ u_{C}\end{array}\right)+\left[\begin{array}{cc}\frac{d}{L} & 0 \\ \frac{d}{L_{B}} & \frac{-1}{L_{B}} \\ 0 & 0\end{array}\right] \cdot\left(\begin{array}{l}u_{1} \\ u_{B}\end{array}\right)+\left[\begin{array}{c}\frac{(d-1)}{L} \\ \frac{(d-1)}{L_{B}} \\ 0\end{array}\right] \cdot u_{V D}$ 
Linearizing this system around the operating point enables us to calculate transfer functions for constructing Bode plots.

The weighed matrix differential equation representing the dynamic behavior of the converter is a nonlinear one. A linearization is necessary to use the possibilities of the linear control theory. We can calculate the linearized small signal model of the converter with capital letters for the operating point values and small letters for the disturbance around the operating point

$$
\begin{array}{ll}
i_{L}=I_{L O}+\hat{i}_{L} & \\
i_{L B}=I_{L B 0}+\hat{i}_{L B} & u_{1}=U_{10}+\hat{u}_{1} . \\
u_{C}=U_{C 0}+\hat{u}_{C} & d=D_{0}+\hat{d}
\end{array}
$$

The following equations can be specified for the stationary (working point) values

$$
\begin{aligned}
& 0=I_{L 0} \cdot\left[-D_{0} \cdot R_{S}+\left(R_{C}+R_{D}\right) \cdot\left(D_{0}-1\right)-R_{L}\right]+ \\
& +I_{L B 0} \cdot\left[-D_{0} \cdot R_{S}+R_{D} \cdot\left(D_{0}-1\right)\right]+ \\
& +U_{C 0} \cdot\left(D_{0}-1\right)+D_{0} \cdot U_{10}+U_{V D} \cdot\left(D_{0}-1\right) \\
& 0=I_{L 0} \cdot\left[-D_{0} \cdot R_{S}+\left(D_{0}-1\right)-R_{D}\right]+ \\
& +I_{L B 0} \cdot\left[-D_{0} \cdot\left(R_{C}+R_{S}\right)-R_{L B}+R_{D} \cdot\left(D_{0}-1\right)\right]+ \\
& +U_{C 0} \cdot D_{0}-U_{B 0}+D_{0} \cdot U_{10}+U_{V D} \cdot\left(D_{0}-1\right) \\
& I_{L B 0}=I_{L 0} \frac{\left(1-D_{0}\right)}{D_{0}} .
\end{aligned}
$$

The same results as derived from the section basic analysis can be achieved in the ideal case (assuming ideal devices with no losses)

$$
\begin{aligned}
& 0=U_{C 0} \cdot\left(D_{0}-1\right)+D_{0} \cdot U_{10} \\
& 0=U_{C 0} \cdot D_{0}-U_{B 0}+D_{0} \cdot U_{10}
\end{aligned}
$$

leading again to

$$
{ }^{B}=\frac{D_{0}}{\left(1-D_{0}\right)} \cdot U_{10} .
$$

One can calculate the linearized small signal model of the converter according to (eq. 42)

\section{Experimental model of the converter}

A small converter model was built. The coils $L$ and $L_{B}$ have about $43 \mu \mathrm{H}$ and a resistor of about $5.6 \mathrm{~m} \Omega$, the capacitor (implemented with an electrolyte capacitor and a film capacitor) has about $100 \mu \mathrm{F}$ and a resistor of about $21 \mathrm{~m} \Omega$. A pulse capacitor is shunted parallel to the electrolyte capacitor for the high frequency component of the capacitor current. The diode has a current rating of $30 \mathrm{~A}$, a differential on-resistance of $30 \mathrm{~m} \Omega$, and a forward voltage of $730 \mathrm{mV}$. The transistor can carry a current of $26 \mathrm{~m} \Omega$ at a maximum drain source voltage of $100 \mathrm{~V}$. The on-resistance is $26 \mathrm{mV}$.

Figure 10 shows the control signal of the active switch for a duty cycle of $50 \%$ (generated by the arbitrary pulse generator),

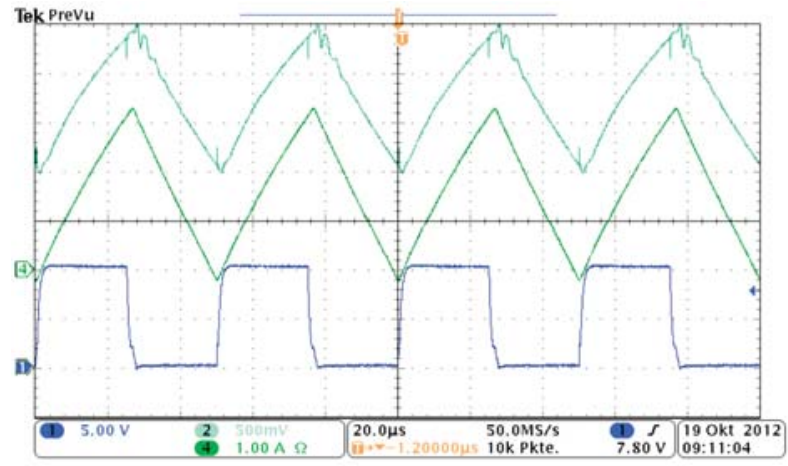

Fig. 10 Gate-source voltage (blue) of the active switch by a duty cycle of 50\%, measurement signal of the LEM current sensor (turquoise) and current through the inductor LB measured by a current probe (green)

$$
\begin{aligned}
& \frac{d}{d t}\left(\begin{array}{l}
\hat{i}_{L} \\
\hat{i}_{L B} \\
\hat{u}_{C}
\end{array}\right)=\left[\begin{array}{ccc}
\frac{-D_{0} \cdot R_{S}+\left(D_{0}-1\right) \cdot\left(R_{C}+R_{D}\right)-R_{L}}{L} & \frac{-\left[D_{0} \cdot R_{S}+\left(1-D_{0}\right) \cdot R_{D}\right]}{L} & \frac{\left(D_{0}-1\right)}{L} \\
\frac{-D_{0} \cdot R_{S}+\left(D_{0}-1\right) \cdot R_{D}}{L_{B}} & \frac{-D_{0} \cdot\left(R_{C}+R_{S}\right)+R_{L B}+\left(1-D_{0}\right) \cdot R_{D}}{L_{B}} & \frac{D_{0}}{L_{B}} \\
\frac{\left(1-D_{0}\right)}{C} & \frac{-D_{0}}{C} & 0
\end{array}\right] . \\
& \left(\begin{array}{l}
\hat{i}_{L} \\
\hat{i}_{L B} \\
\hat{u}_{C}
\end{array}\right)+\left[\begin{array}{l}
\frac{D_{0}}{L} \frac{I_{L 0} \cdot\left(-R_{S}+R_{C}+R_{D}\right)+I_{L B 0} \cdot\left(-R_{S}+R_{D}\right)+U_{C 0}+U_{10}+U_{V D}}{L} \\
\frac{D_{0}}{L_{B}} \frac{I_{L 0} \cdot\left(-R_{S}+R_{D}\right)+I_{L B 0} \cdot\left(-R_{C}-R_{S}+R_{D}\right)+U_{C 0}+U_{10}+U_{V D}}{L_{B}} \\
0
\end{array}\right] \cdot\left(\begin{array}{l}
-I_{L 0}-I_{L B 0} \\
C
\end{array}\right)
\end{aligned}
$$


which is measured at the active switch (blue), the measurement signal of a LEM current sensor (turquoise and triangular shaped) and the current through the inductor $L_{B}$ (green and triangular shaped).

\section{Two-point control}

The two-point control is another control method for controlling the output current of the battery charger. In comparison with the pulse width modulation, the two-point control operates with variable periodic time as well as with variable pulse width. The activation and deactivation of the active switch depends on the instantaneous values of the current to be controlled. The active switch has to be turned off when the current reaches the upper limit and has to be turned on again when the current reaches the lower limit. The difference between the switching points is called hysteresis.

Figure 11 shows the non-inverting Schmitt-trigger. The parallel positive feedback creates the needed hysteresis. The hysteresis voltage is controlled by the proportion between the resistances of $R_{1}$ and $R_{2}$. The resistor $R_{1}$ can be calculated from resistor $R_{2}$, upper threshold voltage $u_{T+}$, lower threshold voltage $u_{T-}$, higher supply voltage $u_{\text {sat }}$ and lower supply voltage $u_{\text {sat }}$ according to

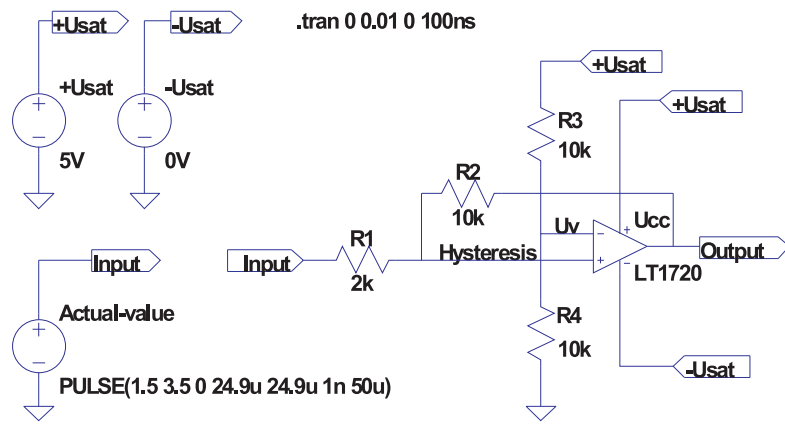

Fig. 11 Non-inverting Schmitt-trigger

$$
R_{1}=\frac{\left(u_{T+}-u_{T-}\right) \cdot R_{2}}{u_{s a t+}-u_{s a t-}} .
$$

The inverting input makes the reference point. The reference voltage is obtained from the voltage divider $R_{3}$ and $R_{4}$. One can calculate the offset voltage $u_{V}$ according to

$$
u_{V}=\frac{u_{T+} \cdot u_{s a t+}-u_{T-} \cdot u_{s a t-}}{u_{s a t+}-u_{s a t-}+u_{T+}-u_{T-}} .
$$

The resistor $R_{4}$ can be calculated from resistor $R_{3}$, offset voltage $u_{V}$ and supply voltage usat according to

$$
R_{1}=\frac{u_{V} \cdot R_{3}}{ \pm u_{s a t}-u_{V}} .
$$

\section{Results of two-point control}

A DC-DC converter with two-point control and current measurement was built for the evaluation of the two-point control in the circuit.

Figure 12 shows the concept. The DC-DC converter with parasitic resistances of the components and fixed forward voltage of the diode as well as the two-point control and current measurement are indicated. A so-called "shunt resistor" RS is indicated for the current measurement. A LEM-current sensor in series with the output inductor is used in the real circuit for current sensing. A driver stage is used in order to turn on or block the MOSFET as quickly as possible.

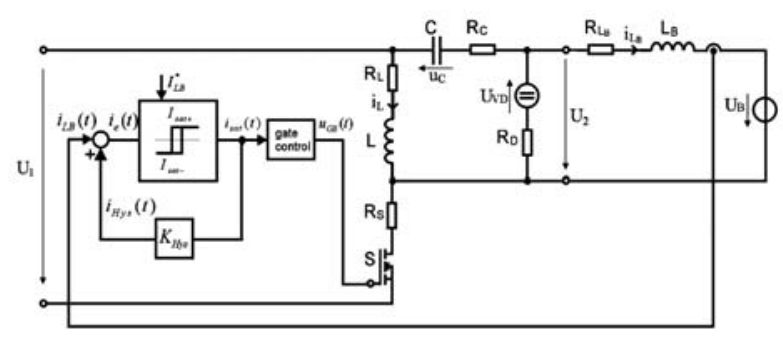

Fig. 12 Converter with two-point control and current measurement

The circuit of the non-inverting Schmitt-trigger is dimensioned so that the upper threshold voltage $u_{T+}$ is and the lower threshold voltage $u_{T-}$ is $2.2 \mathrm{~V}$. The offset voltage $u_{V}$ is $2.4 \mathrm{Vin}$ this case. The supply voltage of the LEM-converter and the gate driver is 10 $\mathrm{V}$. The input voltage of the converter can be up to $36 \mathrm{~V}$.

Figure 13 shows that the control signal (orange) turns to "high", when the measurement signal of the LEM current sensor (red) reaches the lower threshold and it turns to "low", when the measurement signal of the LEM current sensor reaches the upper threshold. The on-time is $6 \mu$ s and the off-time is $17 \mu \mathrm{s}$. This corresponds to a frequency of $59 \mathrm{kHz}$.

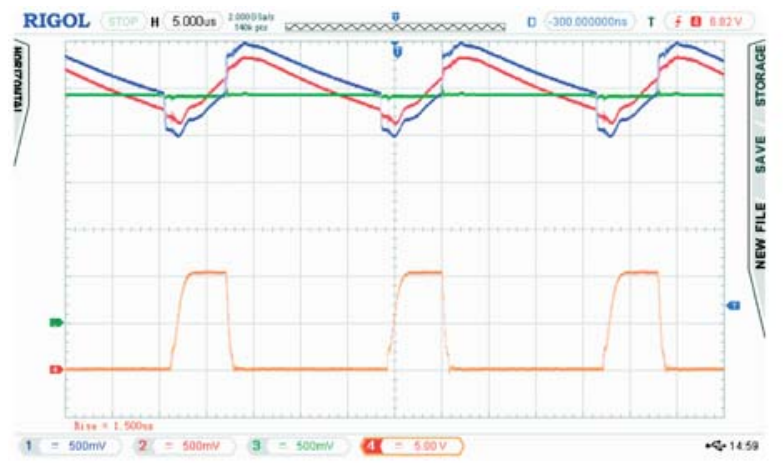

Fig. 13 Measurement signal of the LEM current sensor (red), hysteresis (blue), offset voltage (green) and gate-source voltage (orange) 


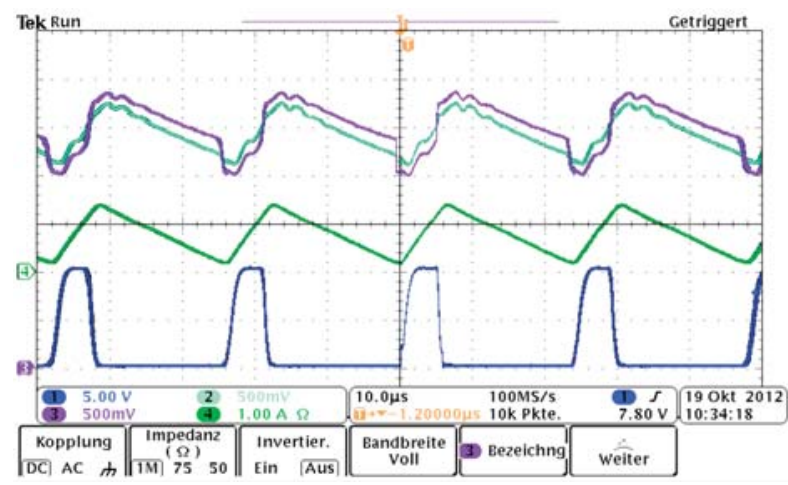

Fig. 14 Measurement signal of the LEM current sensor (turquoise), hysteresis (pink), current through the inductor LB (green) and gate-source voltage (blue)

Figure 14 shows the measurement signal of the LEM current sensor (turquoise), the hysteresis (pink), the current through the inductor $L_{B}$ (green) and the gate-source voltage (blue). From Fig. 10 one can immediately see that the current through the inductor
$L_{B}$ (green) is between $-2.2 \mathrm{~A}$ and $3.3 \mathrm{~V}$. With the two-point control, the current through the inductor $L_{B}$ (green) is limited between 0.2 A to $1.4 \mathrm{~A}$ now.

\section{Conclusion}

Battery chargers are very important, especially for low and medium voltages and low and medium power (e.g. handhelds, cars [8], solar applications [9]). The here treated converter is similar to the basic step-down converter. Due to a simple $L C$ element which is added, the converter generates now a mean voltage across the diode, which can be higher or lower than the input voltage. This is especially useful when only low input voltages are available. The system can be described as a three order system. A twopoint control with a non-inverting two-point controller is useful to control the output voltage or output current. In this way, the active switch is turned on and off. Therefore, it is possible to keep the load current $i_{L B}$ in a defined range by stopping the rise of the coil current via the active switch. The converter is especially applicable for solar and small fuel cell applications. It can be designed also for medium power applications e.g. for charging car batteries out of a solar generator.

\section{References}

[1] HIMMELSTOSS, F.: Ein- und Zweiqudrantenstellglieder, Austrian Patent Office AT 507203 B1 filed 2008.10.30.

[2] HIMMELSTOSS, F. A., FLICKER, Y., RABL, M.: A Simple Two-Quadrant DC Motor Controller, OPTIM 2010.

[3] ZACH, F.: Leistungselektronik, 4. Aufl, Wien : Springer, 2010.

[4] MOHAN, N., UNDELAND, T., ROBBINS, W.: Power Electronics, Converters, Applications and Design (2 ${ }^{\text {nd }}$ Ed.), New York: W. P. John Wiley \& Sons, 2003.

[5] BOLDEA, I., TUTELEA, L.: Electric Machines: Steady State, Transients, and Design with MATLAB, Boca Raton, FL: CRC Press Taylor and Francis Group, 2010.

[6] SCOTARU, P., NICOLAEI, D. V., CERNAT, M., PUKLUS, Z.: Reversible DC-to-DC Converter for a Dual Voltage Automotive System Using Zero Voltage Switching Technique, OPTIM 2008

[7] MARCHESONI, M., VACCA, C.: New DC-DC Converter for Energy Storage System Interfacing in Fuel Cell Hybrid Electric Vehicles, IEEE Trans. On Power Electronics, vol. 54, No. 2, 2007, pp. 301-308.

[8] EMADI, A.: Handbook of Automotive Power Electronics and Motor Drives, Marcel Dekker, 2005.

[9] TURCEK, J., HRASKO, M., ALTUS, J.: Photovoltaics in Present Days and their Coexistence with Power Systems, Communications - Scientific Letters of the University of Zilina, No. 2a, 2011, pp.109-113. 